\title{
PERIODIC SOLUTIONS FOR DYNAMIC EQUATIONS ON TIME SCALES
}

\author{
SHUN-TANG WU AND LONG-YI TSAI
}

\begin{abstract}
The second order nonlinear dynamic system on time scales is considered. Some sufficient conditions for the existence of periodic solutions are given. Differential inequality techniques and the method of mixed monotony are used.
\end{abstract}

\section{Introduction}

In recent years, the initiated theory of dynamic systems on time scales $\mathbb{T}$ (closed subsets of reals) provides a framework for handling both continuous and discrete dynamical systems simultaneously so as to bring out better insight and understanding of subtle differences of these two types of systems. A unified theory is developed by Aulbach and Hilger in [3]. Some integrated results are given by [5], [7] and [10]. Herein some basic properties of solutions of dynamic systems on time scales are introduced. Qualitative properties including Lyapunov stability, oscillation theory and asymptotic behavior of the solutions are also discussed.

The method of upper and lower solutions, coupled with the monotone iterative technique, show itself as an effective mechanism that offer theoretical, as well as constructive existence result in a closed set for differential equations in [9]. The upper and lower solutions are used to generate the closed set served as upper and lower bounds for solutions which can be improved by monotone iterative procedures. Moreover, the iterative schemes are also useful for investigating the qualitative properties of the solutions, hence the method of upper and lower solutions is widely used to discuss the existence, uniqueness, boundedness, stability and asymptotic behavior of the solutions. When the upper solution is assumed to be larger than the lower solution, existence results for nonlinear boundary value problems are given in [8]. For reversing the order of the upper and lower solutions. Some results are given in [6].

Recently the method of upper and lower solutions has been used to discuss dynamic equations on time scales in [2] and [5] by fixed point theorem under the assumption that

Corresponding author: Shun-Tang Wu.

Received February 20, 2008.

2000 Mathematics Subject Classification. 39A11.

Key words and phrases. Periodic solution, dynamic equation, time scales, method of mixed monotony, upper (lower) solutions. 
the upper solution is larger than the lower solution. In this paper we shall consider periodic boundary value problems and employ the method of mixed monotony to obtain extremal solutions under the conditions that the upper solution is greater than or less than the lower solution. The paper is organized as follows. Some preliminaries on dynamic equations are given in Section 2. In Section 3, we first give two lemmas on the existence and uniqueness of solutions for linear periodic problem. These results are used later to discuss the existence of nonlinear periodic problem. The main results are given in Theorem 3.3 and Theorem 3.4. These results will generalize some existence results on periodic boundary value problems $([8]$, $[6$, Section 5.3$])$ on $\mathbb{T}=\mathbb{R}$.

\section{Preliminaries}

We first give some of the basics of the time scale theory and refer to [3] or [5] for further details. Let $\mathbb{T}$ be a time scale which means any closed subset of $\mathbb{R}$. The embedding of $\mathbb{T}$ in $\mathbb{R}$ gives rise to the order and topological structure of the time scale in a canonical way. Since a time scale $\mathbb{T}$ may not be connected, we need the concept of jump operators.

Definition 2.1. The operators $\sigma, \rho: \mathbb{T} \rightarrow \mathbb{T}$ such that

$$
\begin{aligned}
\sigma(t) & =\{\inf \{s \in \mathbb{T}: s>t\}, t \neq \max \mathbb{T}\} \\
\rho(t) & =\{\sup \{s \in \mathbb{T}: s<t\}, t \neq \min \mathbb{T}\}
\end{aligned}
$$

are called forward and backward jump operator respectively. A point $t \in \mathbb{T}$ is called right-scattered, right-dense, left-scattered, and left-dense depending on whether $\sigma(t)>t$, $\sigma(t)=t, \rho(t)>t, \rho(t)=t$, respectively. While the mapping $\mu(t)=\sigma(t)-t$ is called graininess. We denote $u^{\sigma}(t) \equiv u(\sigma(t))$ for $t \in \mathbb{T}$. and $\mathbb{T}^{k}$ be the set of points of $\mathbb{T}$ except for a maximal element which is also left scattered.

Definition 2.2. The mapping $f: \mathbb{T} \rightarrow \mathbb{R}$ is called rd-continuous and denoted by $f \in C_{r d}(\mathbb{T}, \mathbb{R})$ if it is continuous at each right-dense or maximal point $t \in \mathbb{T}$ and if the left-sided limit exists at each left-dense points.

Definition 2.3. Let $f: \mathbb{T} \rightarrow \mathbb{R}$ at each $t \in \mathbb{T}^{k}, f$ has a derivative $f^{\Delta}(t) \in \mathbb{R}$ if for each $\varepsilon>0$, there exists a neighborhood $U$ of $t$ such that,

$$
\left|f(\sigma(t))-f(s)-f^{\Delta}(t)(\sigma(t)-s)\right|<\varepsilon|\sigma(t)-s|, \text { for all } s \in U \cap \mathbb{T}
$$

Definition 2.4. Let $f: \mathbb{T} \rightarrow \mathbb{R}$, we say that $f$ has the second derivative $f^{\Delta \Delta}$ provided that $f^{\Delta}$ is differentiable on $\mathbb{T}^{k^{2}}=\left(\mathbb{T}^{k}\right)^{k}$ with derivative $f^{\Delta \Delta}=\left(f^{\Delta}\right)^{\Delta .} \mathbb{T}^{k^{2}} \rightarrow \mathbb{R}$. Finally, for $t \in \mathbb{T}$, we denote $\sigma^{2}(t)=\sigma(\sigma(t)), \rho^{2}(t)=\rho(\rho(t))$ and $\sigma^{n}(t), \rho^{n}(t)$ for $n \in \mathbb{N}$ are defined accordingly. 


\section{Remark.}

(i) If a function $f: \mathbb{T} \rightarrow \mathbb{R}$ is continuous at a right-scattered point $t$, then it is differentiable at $t$ with derivative

$$
f^{\Delta}(t)=\frac{f(\sigma(t))-f(t)}{\sigma(t)-t} .
$$

(ii) Let $f, g: \mathbb{T}^{k} \rightarrow \mathbb{R}$, and $t \in \mathbb{T}^{k}$. If $f^{\Delta}(t), g^{\Delta}(t)$ exist and $(f g)(t)$ is defined, then $(f g)^{\Delta}(t)$ exists and

$$
(f g)^{\Delta}(t)=f(\sigma(t)) g^{\Delta}(t)+f^{\Delta}(t) g(t) .
$$

Definition 2.5. A mapping $f: \mathbb{T} \rightarrow \mathbb{R}$ is called an antiderivative of $g$ on $\mathbb{T}$ if it is differentiable on $\mathbb{T}$ and satisfies $f^{\Delta}(t)=g(t)$ for $t \in \mathbb{T}^{k}$ and then for any $r, s \in \mathbb{T}^{k}, r \leq s$, we define

$$
\int_{r}^{s} g(t) \Delta t=f(s)-f(r)
$$

as the Cauchy integral of $g$ from $r$ to $s$. Note that for $\mathbb{T}=\mathbb{R}$, the Cauchy integral coincides with the Riemann integral and and that if $g: \mathbb{T}^{k} \rightarrow \mathbb{R}$ is rd-continuous, then $g$ possess the antiderivative.

Lemma 2.6. (Lagrange identity) For $u, v \in C_{r d}^{2}(\mathbb{T}, \mathbb{R})$, we have

$$
v^{\Delta \Delta} u^{\sigma}-u^{\Delta \Delta} v^{\sigma}=W^{\Delta}(u, v),
$$

on $\mathbb{T}^{k^{2}}$. Here the Wronskian $W$ of $u$ and $v$ is defined by $W(u, v)=u v^{\Delta}-v u^{\Delta}$.

Definition 2.7. Let $u$ be a solution of

$$
\left(p(t) u^{\Delta}\right)^{\Delta}+q(t) u^{\sigma}=0, t \in \mathbb{T},
$$

where $p, q \in C_{r d}$ and $p(t) \neq 0$, for $t \in \mathbb{T}$.

(i) We say that $u$ has a generalized zero at $t$ if $u(t)=0$ or if $t$ is left-scattered and $p(\rho(t)) u(\rho(t)) u(t)<0$.

(ii) (2.7) is said to be disconjugate on $[a, b]$, if there is no nontrivial solution of (2.7) with two (or more) generalized zeros in $[a, b]$.

The following lemma is due to Lyapunov inequalities given in [4] or in [5, p.273].

Lemma 2.8. (Sufficient condition for disconjugacy) Let $\mathbb{T} u$ be a solution of the SturmLiouville dynamic equation

$$
u^{\Delta \Delta}+q(t) u^{\sigma}=0, t \in \mathbb{T}
$$

where $q: \mathbb{T} \longrightarrow(0, \infty)$ is positive and rd-continuous. If

$$
\int_{a}^{b} q(t) \Delta t<\frac{b-a}{f(d)},
$$


then (2.8) is discongujate on $[a, b]$, where $f: \mathbb{T} \longrightarrow \mathbb{R}$ is defined by $f(t)=(t-a)(b-t)$, and $d \in \mathbb{T}$ is such that

$$
\left|\frac{a+b}{2}-d\right|=\operatorname{dist}\left(\frac{a+b}{2}, \mathbb{T}\right) .
$$

Definition 2.9. Let $T \in \mathbb{T} \cap[0, \infty)$, we say that a time scale $\mathbb{T}$ is periodic with period $\sigma^{2}(T)$, if for any $t \in \mathbb{T}$, we have $t+\sigma^{2}(T) \in \mathbb{T}$.

Let

$$
A=\left\{u \in C_{p r d}^{1}\left(\left[a, \sigma^{2}(b)\right], R\right) \mid u(a)=u\left(\sigma^{2}(b)\right)=0\right\},
$$

where $C_{p r d}^{1}(I, R)$ denotes the set of all continuous functions whose derivatives are piecewise continuous on $I$, and let

$$
F(u)=\int_{a}^{\sigma^{2}(b)}\left\{\left[u^{\Delta}(t)\right]^{2}-q(t)\left[u(\sigma(t)]^{2}\right\} \Delta t .\right.
$$

Definition 2.10. We say that $F$ is positive definite on $A$ provided that $F(u) \geq 0$ for all $u \in A$ and $F(u)=0$ if and only if $u=0$.

Lemma 2.11.(Jacobi's condition [1]) The equation (2.8) is disconjugate on $\left[a, \sigma^{2}(b)\right]$ if and only if $F$ is positive definite on $A$.

\section{Monotone iterative technique}

Hereafter we shall use the method of upper and lower solutions and monotone iterative technique to investigate the existence of solutions of periodic boundary value problems $(P B V P)$.

Consider the boundary value problem

$$
u^{\Delta \Delta}(t)+f(t, u)=0, t \in \mathbb{T}
$$

with

$$
u(0)=u\left(\sigma^{2}(T)\right), u^{\Delta}(0)=u^{\Delta}(\sigma(T)),
$$

where $f \in C_{r d}\left[\mathbb{T} \times \mathbb{R}^{N}, \mathbb{R}^{N}\right], \mathbb{T}$ is a time scale which is periodic with period $\sigma^{2}(T)$, $T \in \mathbb{T} \cap[0, \infty)$. We shall give two lemmas on the existence and uniqueness of solutions of linear periodic boundary value problems.

Lemma 3.1. (I) The problem

$$
\begin{aligned}
u^{\Delta \Delta}(t)-M^{2} u^{\sigma}(t) & =0, t \in I \cap \mathbb{T} \\
u(0) & =u\left(\sigma^{2}(T)\right) \\
u^{\Delta}(0) & =u^{\Delta}(\sigma(T)),
\end{aligned}
$$

has only trivial solution for $M \in \mathbb{R}-\{0\}$ and $I=\left[0, \sigma^{2}(T)\right]$. 
(II) If $g \in C_{r d}[I \cap \mathbb{T}, \mathbb{R}]$ is nonnegative and nontrivial, then the problem

$$
\begin{aligned}
u^{\Delta \Delta}(t)-M^{2} u^{\sigma}(t) & =g^{\sigma}(t), t \in I \cap \mathbb{T} \\
u(0) & =u\left(\sigma^{2}(T)\right) \\
u^{\Delta}(0) & =u^{\Delta}(\sigma(T))+\lambda,
\end{aligned}
$$

has a unique non-positive solution $u \in C_{r d}^{2}[I \cap \mathbb{T}, \mathbb{R}]$ for $\lambda \geq 0$.

Proof. (I) (i) When $u(0)=u\left(\sigma^{2}(T)\right)=0$, let

$$
F(u)=\int_{0}^{\sigma^{2}(T)}\left\{\left[u^{\Delta}(t)\right]^{2}+M^{2}\left[u(\sigma(t)]^{2}\right\} \Delta t .\right.
$$

We see that $F$ is positive definite on $A$ where $A$ is given in (2.9). Indeed, $F(u) \geq 0$, for all $u \in A$ and if $F(u)=0$, then $u^{\Delta}(t)=0$ and $u(\sigma(t))=0$, for all $t \in\left[0, \sigma^{2}(T)\right]$, hence we have $u(t)=0$ for all $t \in\left[0, \sigma^{2}(T)\right]$. By Lemma 2.11, (3.4) is disconjugate on $\left[0, \sigma^{2}(T)\right]$. Since $u(0)=u\left(\sigma^{2}(T)\right)=0$, by definition of discojugacy, $u(t)=0$, for all $t \in\left[0, \sigma^{2}(T)\right]$.

(ii) When $u(0)=u\left(\sigma^{2}(T)\right)>0$, from (3.3), we have $u^{\Delta}\left(\sigma^{2}(T)\right)>u^{\Delta}(\sigma(T))$. By (3.5), we obtain $u^{\Delta}\left(\sigma^{2}(T)\right)>u^{\Delta}(0)$. But by periodicity of $u$, we have

$$
u^{\Delta}\left(\sigma^{2}(T)\right)=\frac{u\left(\sigma\left(\sigma^{2}(T)\right)-u\left(\sigma^{2}(T)\right)\right.}{\mu\left(\sigma^{2}(T)\right)}=\frac{u(\sigma(0))-u(0)}{\mu(0)}=u^{\Delta}(0),
$$

it leads to a contradiction.

(iii) When $u(0)=u\left(\sigma^{2}(T)\right)<0$, let $v(t)=-u(t)$, we have

$$
\begin{aligned}
v^{\Delta \Delta}(t)-M^{2} v^{\sigma}(t) & =0, t \in I \cap \mathbb{T} \\
v(0) & =v\left(\sigma^{2}(T)\right)>0 \\
v^{\Delta}(0) & =v^{\Delta}(\sigma(T)) .
\end{aligned}
$$

By the same argument as in (ii), we get a contradiction.

$(\mathbf{I I})_{1}$ (Existence and uniqueness). Let $x_{1}$ and $x_{2}$ be two linear independent solutions of the homogeneous equation (3.3). Then for any solution $x$ of (3.3)-(3.5), we have $x(t)=c_{1} x_{1}(t)+c_{2} x(t)$, where $c_{1}$ and $c_{2}$ satisfy the following equation

$$
A\left[\begin{array}{l}
c_{1} \\
c_{2}
\end{array}\right]=\left[\begin{array}{l}
0 \\
0
\end{array}\right]
$$

here

$$
A=\left[\begin{array}{l}
x_{1}(0)-x_{1}\left(\sigma^{2}(T)\right) x_{2}(0)-x_{2}\left(\sigma^{2}(T)\right) \\
x_{1}^{\Delta}(0)-x_{1}^{\Delta}(\sigma(T)) x_{2}^{\Delta}(0)-x_{2}^{\Delta}(\sigma(T))
\end{array}\right]
$$


Since the only solution of (3.3), (3.4), (3.5) is trivial solution, so $c_{1}=c_{2}=0$, thus $\operatorname{det} A \neq 0$. Let $u_{0}$ be a particular solution of (3.6). Then any solution of (3.6)-(3.8) is given by

$$
u(t)=a_{1} x_{1}(t)+a_{2} x_{2}(t)+u_{0}(t),
$$

for some $a_{1}$ and $a_{2}$ and satisfies

$$
A\left[\begin{array}{l}
a_{1} \\
a_{2}
\end{array}\right]=\left[\begin{array}{c}
-u_{0}(0)+u_{0}\left(\sigma^{2}(T)\right) \\
-u_{0}(0)+u_{0}(\sigma(T))+\lambda
\end{array}\right] .
$$

Since $\operatorname{det} A \neq 0$, so (3.6) -(3.8) has only a unique solution.

$(\mathbf{I I})_{2}$ Next we claim that $u \leq 0$ on $I \cap \mathbb{T}$. Suppose not, then $u$ has a positive maximum in $\left[0, \sigma^{2}(T)\right]$, hence there exists a maximum point $c$ in $\left[0, \sigma^{2}(T)\right]$.

(i) If $c \in\left(0, \sigma^{2}(T)\right)$, then $u(t)<u(c)$ for $t \in\left(c, \sigma^{2}(T)\right]$. Consider the following cases :

Case $1: \rho(c)=c<\sigma(c)(c$ is right-scattered and left-dense). Since

$$
\lim _{t \rightarrow c^{-}} u^{\Delta}(t)=u^{\Delta}(c)=\frac{u(\sigma(c))-u(c)}{\mu(c)}<0,
$$

hence there exists $\delta>0$ such that $u^{\Delta}(t)<0$ on $(c-\delta, c]$. This implies $u(t)$ is decreasing on $(c-\delta, c]$, which contradicts the way $c$ was chosen.

Case $2: \rho(c)<c<\sigma(c)$ ( $c$ is isolated). Note that

$$
u^{\Delta}(c)=\frac{u(\sigma(c))-u(c)}{\mu(c)}<0,
$$

and

$$
u^{\Delta \Delta}(\rho(c))=\frac{u^{\Delta}(c)-u^{\Delta}(\rho(c))}{\mu(\rho(c))} .
$$

This implies $u^{\Delta \Delta}(\rho(c))<0$.

Case 3: $\rho(c)<c=\sigma(c)$ (c is right-dense and left-scattered). If $u^{\Delta}(c)>0$, then $\lim _{t \rightarrow c^{+}} u^{\Delta}(t)=u^{\Delta}(c)>0$, hence there exists $\delta>0$ such that $u^{\Delta}(t)>0$ on $[c, c+\delta)$. This implies $u(t)$ is increasing on $[c, c+\delta)$, which contradicts the way $c$ was chosen. Therefore $u^{\Delta}(c) \leq 0$. Since $\rho(c)$ is right-scattered,

$$
u^{\Delta \Delta}(\rho(c))=\frac{u^{\Delta}(c)-u^{\Delta}(\rho(c))}{\mu(\rho(c))}<0 .
$$

Case $4: \rho(c)=c=\sigma(c)$ ( $c$ is dense). As in Case 3, we have $u^{\Delta}(c) \leq 0$. Suppose that $u^{\Delta}(c)<0$, then

$$
\lim _{t \rightarrow c} u^{\Delta}(t)=u^{\Delta}(c)<0
$$


and there exists $\delta>0$ such that $u^{\Delta}(t)<0$ on $(c-\delta, c]$. Hence $u(t)$ is decreasing on $(c-\delta, c]$ that contradicts the way $c$ was chosen. Thus $u^{\Delta}(c)=0$. Suppose that $u^{\Delta \Delta}(\rho(c))>0$, since $c$ is right-dence, and $u \in C_{r d}^{2}$, there exists $\delta>0$ such that $u^{\Delta}(t)>0$ on $[c, c+\delta)$. That means $u^{\Delta}$ is strictly increasing on $[c, c+\delta)$. Since $u^{\Delta}(c)=0, u^{\Delta}(t)>0$ on $(c, c+\delta)$. This implies $u(t)$ is increasing on $(c, c+\delta)$, which contradicts the way $c$ was chosen. Hence $u^{\Delta \Delta}(\rho(c)) \leq 0$. From Case $2-4$, we conclude that $u^{\Delta \Delta}(\rho(c)) \leq 0$. On the other hand, from (3.6), we have

$$
u^{\Delta \Delta}(\rho(c))=M^{2} u(c)+g(c)>0 .
$$

It contradicts to the fact $u^{\Delta \Delta}(\rho(c)) \leq 0$.

(ii) If $c=0$ or $c=\sigma^{2}(T)$, then $u^{\Delta}(0) \leq 0$ and $u^{\Delta}(\sigma(T)) \geq 0$. By (3.7) and (3.8), we have

$$
u^{\Delta}(0)=u^{\Delta}(\sigma(T))=0 .
$$

Hence $u(0)=u(\sigma(0))>0$. Since

$$
u^{\Delta \Delta}(0)=M^{2} u^{\sigma}(0)+g^{\sigma}(0) \geq M^{2} u^{\sigma}(0)>0,
$$

$u^{\Delta}(\sigma(0))>u^{\Delta}(0)=0$. This implies $u\left(\sigma^{2}(0)\right)>u(\sigma(0))=u(0)$, it leads to a contradiction, since $u$ has a maximum at 0 . Another variant is the following result which generalizes a lemma of [6] to the time scales.

Lemma 3.2.(I) The problem

$$
\begin{aligned}
u^{\Delta \Delta}(t)+k u^{\sigma}(t) & =0, t \in I \cap \mathbb{T} \\
u(0) & =u\left(\sigma^{2}(T)\right) \\
u^{\Delta}(0) & =u^{\Delta}(\sigma(T)),
\end{aligned}
$$

has only trivial solution if

$$
0<k<\frac{1}{d\left(\sigma^{2}(T)-d\right)}
$$

where $d \in \mathbb{T}$ is given by

$$
\left|\frac{\sigma^{2}(T)}{2}-d\right|=\operatorname{dist}\left(\frac{\sigma^{2}(T)}{2}, \mathbb{T}\right)
$$

(II) If $0<k \leq k^{*}$, where

$$
k^{*}=\min \left\{\frac{1}{d\left(\sigma^{2}(T)-d\right)}, \frac{1}{L \sigma^{2}(T)}\right\},
$$

and $L$ is given in (3.19), and if $g \in C_{r d}[I \cap \mathbb{T}, \mathbb{R}]$ is nonnegative and nontrivial, then the solution $u \in C_{r d}^{2}[I \cap \mathbb{T}, \mathbb{R}]$ of the problem

$$
\begin{aligned}
u^{\Delta \Delta}(t)+k u^{\sigma}(t) & =g^{\sigma}(t), t \in I \cap \mathbb{T} \\
u(0) & =u\left(\sigma^{2}(T)\right) \geq 0 \\
u^{\Delta}(0) & =u^{\Delta}(\sigma(T))+\lambda,
\end{aligned}
$$


is nonnegative on $I \cap \mathbb{T}$ for any $\lambda \geq 0$.

Proof. (I) (i) When $u(0)=u\left(\sigma^{2}(T)\right)=0$, by Lemma 2.11, (3.9) is disconjugate on $\left[0, \sigma^{2}(T)\right]$. Since $u(0)=u\left(\sigma^{2}(T)\right)=0$, by definition of disconjugacy, we have $u(t) \equiv 0$, on $\left[0, \sigma^{2}(T)\right]$.

(ii) When $u(0)=u\left(\sigma^{2}(T)\right)>0$, from (3.9), $u^{\Delta \Delta}(\sigma(T))<0$. This implies that $u^{\Delta}\left(\sigma^{2}(T)\right)<u^{\Delta}(\sigma(T))$. By (3.11), we have $u^{\Delta}\left(\sigma^{2}(T)\right)<u^{\Delta}(0)$. On the other hand, by periodicity of $u$, we have

$$
u^{\Delta}\left(\sigma^{2}(T)\right)=\frac{u\left(\sigma\left(\sigma^{2}(T)\right)-u\left(\sigma^{2}(T)\right)\right.}{\mu\left(\sigma^{2}(T)\right)}=\frac{u(\sigma(0))-u(0)}{\mu(0)}=u^{\Delta}(0),
$$

it leads to a contradiction.

(iii) When $u(0)=u\left(\sigma^{2}(T)\right)<0$, let $v(t)=-u(t)$, we have

$$
\begin{aligned}
v^{\Delta \Delta}(t)+k v^{\sigma}(t) & =0, t \in I \cap \mathbb{T}, \\
v(0) & =v\left(\sigma^{2}(T)\right)>0, \\
v^{\Delta}(0) & =v^{\Delta}(\sigma(T)) .
\end{aligned}
$$

By the same argument as in (ii), we get a contradiction.

(II) Case 1: $u(0)=u\left(\sigma^{2}(T)\right)>0$.

(i) If there exists some $t^{*} \in \mathbb{T}$ such that $u\left(t^{*}\right)>0$ and $u\left(\sigma\left(t^{*}\right)\right)<0$. Let $v(t)$ be a solution of the following problem

$$
\begin{aligned}
v^{\Delta \Delta}(t)+k v^{\sigma}(t) & =0, t \in\left(\sigma\left(t^{*}\right), \sigma\left(t^{*}\right)+\sigma^{2}(T)\right] \\
v\left(\sigma\left(t^{*}\right)\right) & =0, v^{\Delta}\left(\sigma\left(t^{*}\right)\right)=1 .
\end{aligned}
$$

Then (3.17)-(3.18) has a unique solution on $\left[\sigma\left(t^{*}\right), \sigma\left(t^{*}\right)+\sigma^{2}(T)\right]$. By (3.12), (3.13) and Lemma $2.11,(3.17)$ is disconjugate, so $v(t)>0$ on $\left(\sigma\left(t^{*}\right), \sigma\left(t^{*}\right)+\sigma^{2}(T)\right]$. Since $v \in C_{r d}^{2}$ on $\left[\sigma\left(t^{*}\right), \sigma\left(t^{*}\right)+\sigma^{2}(T)\right]$, we put

$$
L=\sup _{t \in\left[\sigma\left(t^{*}\right), \sigma\left(t^{*}\right)+\sigma^{2}(T)\right]}|v(t)| .
$$

By (3.13), we have

$$
\begin{aligned}
v^{\Delta}\left(t^{*}+\sigma^{2}(T)\right) & =v^{\Delta}\left(\sigma\left(t^{*}\right)\right)-k \int_{\sigma\left(t^{*}\right)}^{t^{*}+\sigma^{2}(T)} v^{\sigma} \Delta s \\
& \geq v^{\Delta}\left(\sigma\left(t^{*}\right)\right)-k^{*} \int_{\sigma\left(t^{*}\right)}^{t^{*}+\sigma^{2}(T)} v^{\sigma} \Delta s \\
& \geq 1-k^{*} L \sigma^{2}(T) \geq 0 .
\end{aligned}
$$


By Lemma 2.6 and (3.18), we have

$$
\begin{aligned}
& \int_{\sigma\left(t^{*}\right)}^{t^{*}+\sigma^{2}(T)}\left(u^{\Delta \Delta} v^{\sigma}-v^{\Delta \Delta} u^{\sigma}\right) \Delta s \\
= & \left(u^{\Delta} v-v^{\Delta} u\right)\left(t^{*}+\sigma^{2}(T)\right)-\left(u^{\Delta} v-v^{\Delta} u\right)\left(\sigma\left(t^{*}\right)\right) \\
= & u^{\Delta}\left(t^{*}\right) v\left(t^{*}+\sigma^{2}(T)\right)-v^{\Delta}\left(t^{*}+\sigma^{2}(T)\right) u\left(t^{*}\right)+v^{\Delta}\left(\sigma\left(t^{*}\right)\right) u\left(\sigma\left(t^{*}\right)\right) .
\end{aligned}
$$

On the other hand, by (3.14) and (3.17), we have

$$
\int_{\sigma\left(t^{*}\right)}^{t^{*}+\sigma^{2}(T)}\left(u^{\Delta \Delta} v^{\sigma}-v^{\Delta \Delta} u^{\sigma}\right) \Delta s=\int_{\sigma\left(t^{*}\right)}^{t^{*}+\sigma^{2}(T)} g^{\sigma}(s) v^{\sigma}(s) \Delta s>0 .
$$

Thus by (3.20), (3.21) and (3.22), we get

$$
u^{\Delta}\left(t^{*}\right) v\left(t^{*}+\sigma^{2}(T)\right)>v^{\Delta}\left(t^{*}+\sigma^{2}(T)\right) u\left(t^{*}\right)-v^{\Delta}\left(\sigma\left(t^{*}\right)\right) u\left(\sigma\left(t^{*}\right)\right)>0 .
$$

Since $v\left(t^{*}+\sigma^{2}(T)\right)>0$, hence $u^{\Delta}\left(t^{*}\right)>0$. It contradicts to the assumption $u\left(t^{*}\right)>0$ and $u\left(\sigma\left(t^{*}\right)\right)<0$.

(ii) If there exists some $t^{*} \in \mathbb{T}$ such that $u\left(t^{*}\right)=0$. Let $v(t)$ be a solution of the following problem

$$
\begin{aligned}
v^{\Delta \Delta}(t)+k v^{\sigma}(t) & =0, t \in\left(t^{*}, t^{*}+\sigma^{2}(T)\right] \\
v\left(t^{*}\right) & =0, v^{\Delta}\left(t^{*}\right)=1 .
\end{aligned}
$$

Then $(3.23)-(3.24)$ has a unique solution on $\left[t^{*}, t^{*}+\sigma^{2}(T)\right]$ and $v(t)>0$ on $\left(t^{*}, t^{*}+\right.$ $\left.\sigma^{2}(T)\right]$. By Lemma 2.6, we have

$$
\begin{aligned}
& \int_{t^{*}}^{t^{*}+\sigma^{2}(T)}\left(u^{\Delta \Delta} v^{\sigma}-v^{\Delta \Delta} u^{\sigma}\right) \Delta s \\
= & \left(u^{\Delta} v-v^{\Delta} u\right)\left(t^{*}+\sigma^{2}(T)\right)-\left(u^{\Delta} v-v^{\Delta} u\right)\left(t^{*}\right) \\
= & u^{\Delta}\left(t^{*}\right) v\left(t^{*}+\sigma^{2}(T)\right) .
\end{aligned}
$$

On the other hand, by (3.14) and (3.23), we obtain

$$
\int_{t^{*}}^{t^{*}+\sigma^{2}(T)}\left(u^{\Delta \Delta} v^{\sigma}-v^{\Delta \Delta} u^{\sigma}\right) \Delta s=\int_{t^{*}}^{t^{*}+\sigma^{2}(T)} g^{\sigma}(s) v^{\sigma}(s) \Delta s>0
$$

Hence from (3.25), (3.26), we have

$$
u^{\Delta}\left(t^{*}\right) v\left(t^{*}+\sigma^{2}(T)\right)>0 .
$$

Since $v\left(t^{*}+\sigma^{2}(T)\right)>0$, hence $u^{\Delta}\left(t^{*}\right)>0$. So $u\left(\sigma\left(t^{*}\right)\right)>0$. Therefore from $(i),(i i)$, we have $u(t) \geq 0, t \in\left[0, \sigma^{2}(T)\right]$. 
Case 2: $u(0)=u\left(\sigma^{2}(T)\right)=0$

(i) If $u(t)<0$ for all $t \in\left(0, \sigma^{2}(T)\right)$, then $u^{\Delta}(0)<0$ and $u^{\Delta}(\sigma(T))>0$. It contradicts to $(3.16)$.

(ii) If there exists $t^{*} \in\left(0, \sigma^{2}(T)\right)$ such that $u\left(t^{*}\right)=0$ and $u(t)<0, t \in\left(0, t^{*}\right)$. Consider

$$
\begin{aligned}
v^{\Delta \Delta}(t)+k v^{\sigma}(t) & =0, t \in\left(t^{*}, t^{*}+\sigma^{2}(T)\right] \\
v\left(t^{*}\right) & =0, v^{\Delta}\left(t^{*}\right)=-1 .
\end{aligned}
$$

By the similar arguments of Case 1 in (ii), we get $u^{\Delta}\left(t^{*}\right)>0$. So $u\left(\sigma\left(t^{*}\right)\right)>0$. Next we consider

$$
\begin{aligned}
u^{\Delta \Delta}(t)+k u^{\sigma}(t) & =g^{\sigma}(t), t \in\left(\sigma\left(t^{*}\right), \sigma\left(t^{*}\right)+\sigma^{2}(T)\right] \\
u\left(\sigma\left(t^{*}\right)\right) & =u\left(\sigma\left(t^{*}\right)+\sigma^{2}(T)\right) \\
u^{\Delta}\left(\sigma\left(t^{*}\right)\right) & =u^{\Delta}\left(\sigma\left(t^{*}\right)+\sigma(T)\right) .
\end{aligned}
$$

If there exists $t_{1} \in\left(\sigma\left(t^{*}\right), t^{*}+\sigma^{2}(T)\right)$ such that $u\left(t_{1}\right)=0$, by Case $1, u^{\Delta}\left(t_{1}\right)>0$. Now $u\left(\sigma^{2}(T)\right)=0$, we have $u^{\Delta}\left(\sigma^{2}(T)\right)>0$. This implies that $u\left(\sigma\left(\sigma^{2}(T)\right)\right)>0$. But $u\left(\sigma\left(\sigma^{2}(T)\right)\right)=u(\sigma(0))<0$. It leads to a contradiction.

(iii) If there exists some $t^{*} \in\left(0, \sigma^{2}(T)\right)$ such that $u\left(\sigma\left(t^{*}\right)\right)>0$ and $u(t)<0, t \in$ $\left(0, t^{*}\right]$. Consider

$$
\begin{aligned}
u^{\Delta \Delta}(t)+k u^{\sigma}(t) & =g^{\sigma}(t), t \in\left(\sigma\left(t^{*}\right), \sigma\left(t^{*}\right)+\sigma^{2}(T)\right] \\
u\left(\sigma\left(t^{*}\right)\right) & =u\left(\sigma\left(t^{*}\right)+\sigma^{2}(T)\right) \\
u^{\Delta}\left(\sigma\left(t^{*}\right)\right) & =u^{\Delta}\left(\sigma\left(t^{*}\right)+\sigma(T)\right) .
\end{aligned}
$$

Since $u\left(\sigma\left(t^{*}\right)\right)=u\left(\sigma\left(t^{*}\right)+\sigma^{2}(T)\right)>0$. Then by Case 1 , we get $u^{\Delta}\left(\sigma^{2}(T)\right)>0$.This implies $u\left(\sigma\left(\sigma^{2}(T)\right)\right)>0$. But $u\left(\sigma\left(\sigma^{2}(T)\right)\right)=u(\sigma(0))<0$. It is a contradiction.

By Case 1 and Case 2, we see that $u(t) \geq 0, t \in\left[0, \sigma^{2}(T)\right] \cap \mathbb{T}$. Hereafter, we shall prove several existence results under the assumptions that upper solution is greater or less than lower solution. The method of mixed monotony ([9]) is used.

Theorem 3.3. Assume that there exist $F \in C\left(I \times \mathbb{R}^{N} \times \mathbb{R}^{N}, \mathbb{R}^{N}\right)$, a positive constant $\varepsilon$, and two functions $\alpha, \beta \in C_{r d}^{1}\left(I \cap \mathbb{T}, \mathbb{R}^{N}\right)$ satisfying the following conditions :

(A1) $\alpha(t) \leq \beta(t), t \in I \cap \mathbb{T}$,

(A2) for all $u, v \in C\left(I \cap \mathbb{T}, \mathbb{R}^{N}\right)$ with $\alpha \leq u \leq \beta$ and $\alpha \leq v \leq \beta$, we have

$$
\begin{gathered}
\alpha_{i}^{\Delta \Delta}+F_{i}(t, u, v) \geq-\frac{1}{2 \varepsilon}\left[\left(v_{i}^{\sigma}-\alpha_{i}^{\sigma}\right)+\left(u_{i}^{\sigma}-\alpha_{i}^{\sigma}\right)\right], \\
\alpha(0)=\alpha\left(\sigma^{2}(T)\right), \quad \alpha^{\Delta}(0) \geq \alpha^{\Delta}(\sigma(T)) .
\end{gathered}
$$

(A3) for all $u, v \in C\left(I \cap \mathbb{T}, \mathbb{R}^{N}\right)$ with $\alpha \leq u \leq \beta$ and $\alpha \leq v \leq \beta$, we have

$$
\begin{gathered}
\beta_{i}^{\Delta \Delta}+F_{i}(t, u, v) \leq-\frac{1}{2 \varepsilon}\left[\left(v_{i}^{\sigma}-\beta_{i}^{\sigma}\right)+\left(u_{i}^{\sigma}-\beta_{i}^{\sigma}\right)\right], \\
\beta(0)=\beta\left(\sigma^{2}(T)\right), \beta^{\Delta}(0) \leq \beta^{\Delta}(\sigma(T)) .
\end{gathered}
$$


(A4) $F_{i}(t, u, v)$ is nondecreasing in $u$ and monotone non-increasing in $v$.

(A5) $F_{i}(t, u, u)=f_{i}(t, u), 1 \leq i \leq N$.

(A6) If there exist two functions $\rho, \gamma \in C_{r d}^{1}\left(I \cap \mathbb{T}, \mathbb{R}^{N}\right)$ such that

$$
\begin{gathered}
\rho_{i}^{\Delta \Delta}+F_{i}(t, \rho, \gamma)=-\frac{1}{2 \varepsilon}\left(\gamma_{i}^{\sigma}-\rho_{i}^{\sigma}\right), \\
\rho(0)=\rho\left(\sigma^{2}(T)\right), \rho^{\Delta}(0)=\rho^{\Delta}(\sigma(T)),
\end{gathered}
$$

and

$$
\begin{gathered}
\gamma_{i}^{\Delta \Delta}+F_{i}(t, \gamma, \rho)=-\frac{1}{2 \varepsilon}\left(\rho_{i}^{\sigma}-\gamma_{i}^{\sigma}\right), \\
\gamma(0)=\gamma\left(\sigma^{2}(T)\right), \gamma^{\Delta}(0)=\gamma^{\Delta}(\sigma(T)),
\end{gathered}
$$

then $\rho \equiv \gamma$ on $I \cap \mathbb{T}$. Thus the problem (3.1), (3.2) has a unique solution $u(t)$ with $\alpha(t) \leq u(t) \leq \beta(t)$ on $I \cap \mathbb{T}$.

Proof. For a pair $(\eta, \tau) \in[\alpha, \beta] \times[\alpha, \beta]$ with $\eta \leq \tau$, consider the linear boundary value problems :

$$
\begin{aligned}
& u_{i}^{\Delta \Delta}-\frac{1}{\varepsilon} u_{i}^{\sigma}=-F_{i}(t, \eta, \tau)-\frac{1}{2 \varepsilon}\left[\eta_{i}^{\sigma}+\tau_{i}^{\sigma}\right] \\
& u(0)=u\left(\sigma^{2}(T)\right), u^{\Delta}(0)=u^{\Delta}(\sigma(T)),
\end{aligned}
$$

and

$$
\begin{aligned}
& w_{i}^{\Delta \Delta}-\frac{1}{\varepsilon} w_{i}^{\sigma}=-F_{i}(t, \tau, \eta)-\frac{1}{2 \varepsilon}\left[\eta_{i}^{\sigma}+\tau_{i}^{\sigma}\right] \\
& w(0)=w\left(\sigma^{2}(T)\right), w^{\Delta}(0)=w^{\Delta}(\sigma(T)) .
\end{aligned}
$$

Let $V=u-w$, we have

$$
\begin{gathered}
V_{i}^{\Delta \Delta}-\frac{1}{\varepsilon} V_{i}^{\sigma}=F_{i}(t, \tau, \eta)-F_{i}(t, \eta, \tau) \\
V(0)=V\left(\sigma^{2}(T)\right), V^{\Delta}(0)=V^{\Delta}(\sigma(T)) .
\end{gathered}
$$

By the mixed monotonicity of $F$ in (A4), the right hand side of the equation is nonnegative. Hence by Lemma 3.1, we get $V \leq 0$ on $I \cap \mathbb{T}$. Hence we have $u \leq w$ on $I \cap \mathbb{T}$. Also we see that $w \leq \beta$ on $I \cap \mathbb{T}$. In fact, let $W=w-\beta$ on $I \cap \mathbb{T}$, we have

$$
\begin{gathered}
W_{i}^{\Delta \Delta}-\frac{1}{\varepsilon} W_{i}^{\sigma} \geq 0 \\
W(0)=W\left(\sigma^{2}(T)\right), W^{\Delta}(0) \geq W^{\Delta}(\sigma(T)) .
\end{gathered}
$$

By Lemma 3.1 again, we have $w \leq \beta$ on $I \cap \mathbb{T}$. Similarly we have $\alpha \leq u$ on $I \cap \mathbb{T}$. Thus we conclude that there exists a unique solution $u$ of (3.31) and unique solution $w$ of (3.32) such that $\alpha \leq u \leq w \leq \beta$ on $I \cap \mathbb{T}$. We then define a map $\Psi$ from $C\left(I \cap \mathbb{T}, R^{2 N}\right)$ into itself by $\Psi(\eta, \tau)=(u, w)$. Note that $\Psi$ is continuous and compact. If we start from $\left(\alpha^{0}, \beta^{0}\right)=(\alpha, \beta)$, let $\left(\alpha^{n+1}, \beta^{n+1}\right)=\Psi\left(\alpha^{n}, \beta^{n}\right)$, for $n \geq 0$. We generate two sequences of functions, $\left\{\alpha^{n}\right\}$ and $\left\{\beta^{n}\right\}$ such that

$$
\alpha \leq \alpha^{1} \leq \cdots \leq \alpha^{n} \leq \cdots \leq \beta^{n} \leq \cdots \leq \beta^{1} \leq \beta,
$$


on $I \cap \mathbb{T}$. Since $\left\{\alpha^{n}\right\}$ and $\left\{\beta^{n}\right\}$ are uniformly bounded, we then have two convergent subsequences $\left\{\alpha^{n_{k}}\right\}$ and $\left\{\beta^{n_{k}}\right\}$ with $\alpha^{n_{k}} \rightarrow \alpha^{*}$ and $\beta^{n_{k}} \rightarrow \beta_{*}$ uniformly on $I \cap \mathbb{T}$, as $n_{k} \rightarrow \infty$. By monotonicity of $\left\{\beta^{n}\right\}$ and $\left\{\alpha^{n}\right\}$, we see that $\beta^{n} \rightarrow \beta_{*}$ and $\alpha^{n} \rightarrow \alpha^{*}$ as $n \rightarrow \infty$. Thus we obtain $\Psi\left(\alpha^{*}, \beta_{*}\right)=\left(\alpha^{*}, \beta_{*}\right)$. By (A6), $\beta_{*} \equiv \alpha^{*}$ on $I \cap \mathbb{T}$. By (A5), we see that $\alpha^{*}$ is a solution of (3.1), (3.2).

Theorem 3.4. Assume that there exists $F \in C\left(I \times \mathbb{R}^{N} \times \mathbb{R}^{N}, \mathbb{R}^{N}\right)$, and two functions $\alpha, \beta \in C_{r d}^{1}\left(I, \mathbb{R}^{N}\right)$ satisfying the following conditions :

(B1) $\beta(t) \leq \alpha(t)$ on $I \cap \mathbb{T}$,

(B2) for all $u, v \in C\left(I \cap \mathbb{T}, \mathbb{R}^{N}\right)$ with $\beta \leq u \leq \alpha$ and $\beta \leq v \leq \alpha$, we have

$$
\begin{gathered}
\alpha_{i}^{\Delta \Delta}+F_{i}(t, u, v) \geq \frac{k^{*}}{2}\left[\left(v_{i}^{\sigma}-\alpha_{i}^{\sigma}\right)+\left(u_{i}^{\sigma}-\alpha_{i}^{\sigma}\right)\right] \\
\alpha(0)=\alpha\left(\sigma^{2}(T)\right), \alpha^{\Delta}(0) \geq \alpha^{\Delta}(\sigma(T)) .
\end{gathered}
$$

where $k^{*}$ is given in Lemma 3.2 .

(B3) for all $u, v \in C\left(I \cap \mathbb{T}, \mathbb{R}^{N}\right)$ with $\beta \leq u \leq \alpha$ and $\beta \leq v \leq \alpha$, we have

$$
\begin{gathered}
\beta_{i}^{\Delta \Delta}+F_{i}(t, u, v) \leq \frac{k^{*}}{2}\left[\left(v_{i}^{\sigma}-\beta_{i}^{\sigma}\right)+\left(u_{i}^{\sigma}-\beta_{i}^{\sigma}\right)\right] \\
\beta(0)=\beta\left(\sigma^{2}(T)\right), \beta^{\Delta}(0) \leq \beta^{\Delta}(\sigma(T)) .
\end{gathered}
$$

(B4) $F_{i}(t, u, v)$ is non-increasing in $u$ and non-decreasing in $v$.

(B5) $F_{i}(t, u, u)=f_{i}(t, u), 1 \leq i \leq N$.

(B6) If there exist two functions $\rho, \gamma \in C_{r d}^{1}\left(I \cap \mathbb{T}, \mathbb{R}^{N}\right)$ such that

$$
\begin{gathered}
\rho_{i}^{\Delta \Delta}+F_{i}(t, \rho, \gamma)=\frac{k^{*}}{2}\left(\gamma_{i}^{\sigma}-\rho_{i}^{\sigma}\right) \\
\rho(0)=\rho\left(\sigma^{2}(T)\right), \quad \rho^{\Delta}(0)=\rho^{\Delta}(\sigma(T)),
\end{gathered}
$$

and

$$
\begin{gathered}
\gamma_{i}^{\Delta \Delta}+F_{i}(t, \gamma, \rho)=\frac{k^{*}}{2}\left(\rho_{i}^{\sigma}-\gamma_{i}^{\sigma}\right), \\
\gamma(0)=\gamma\left(\sigma^{2}(T)\right), \quad \gamma^{\Delta}(0)=\gamma^{\Delta}(\sigma(T)),
\end{gathered}
$$

then $\rho \equiv \gamma$ on $I \cap \mathbb{T}$.

Then the problem (3.1), (3.2) has a unique solution $u(t)$ with $\beta(t) \leq u(t) \leq \alpha(t)$ on $I \cap \mathbb{T}$.

Proof. For a pair $(\eta, \tau) \in[\beta, \alpha] \times[\beta, \alpha]$ with $\eta \leq \tau$, consider the linear boundary value problems :

$$
\begin{gathered}
u_{i}^{\Delta \Delta}+k^{*} u_{i}^{\sigma}=-F_{i}(t, \eta, \tau)+\frac{k^{*}}{2}\left[\eta_{i}^{\sigma}+\tau_{i}^{\sigma}\right] \\
u(0)=u\left(\sigma^{2}(T)\right), u^{\Delta}(0)=u^{\Delta}(\sigma(T)),
\end{gathered}
$$

and

$$
\begin{aligned}
& w_{i}^{\Delta \Delta}+k^{*} w_{i}^{\sigma}=-F_{i}(t, \tau, \eta)+\frac{k^{*}}{2}\left[\eta_{i}^{\sigma}+\tau_{i}^{\sigma}\right] \\
& w(0)=w\left(\sigma^{2}(T)\right), \quad w^{\Delta}(0)=w^{\Delta}(\sigma(T)) .
\end{aligned}
$$


Let $V=w-u$, we have

$$
\begin{gathered}
V_{i}^{\Delta \Delta}+k^{*} V_{i}^{\sigma}=F_{i}(t, \eta, \tau)-F_{i}(t, \tau, \eta) \\
V(0)=V\left(\sigma^{2}(T)\right), \quad V^{\Delta}(0)=V^{\Delta}(\sigma(T)) .
\end{gathered}
$$

By the mixed monotonicity of $F$ in (B4), the right hand side of the equation is nonnegative. Hence by Lemma 3.2, we get $V \geq 0$ on $I \cap \mathbb{T}$. Hence we have $w \geq u$ on $I \cap \mathbb{T}$. Also we see that $u \geq \beta$ on $I \cap \mathbb{T}$. In fact, let $W=u-\beta$ on $I \cap \mathbb{T}$, we have

$$
\begin{gathered}
W_{i}^{\Delta \Delta}+k^{*} W_{i}^{\sigma} \geq 0 \\
W(0)=W\left(\sigma^{2}(T)\right), \quad W^{\Delta}(0) \geq W^{\Delta}(\sigma(T)) .
\end{gathered}
$$

By Lemma 3.2 again, we have $u \geq \beta$ on $I \cap \mathbb{T}$. Similarly we have $\alpha \geq w$ on $I \cap \mathbb{T}$. Thus we conclude that there exists a unique solution $u$ of (3.39) and unique solution $w$ of (3.40) such that $\alpha \geq w \geq u \geq \beta$ on $I \cap \mathbb{T}$. We then define a map $\Psi$ from $C\left(I \cap \mathbb{T}, R^{2 N}\right)$ into itself by $\Psi(\eta, \tau)=(u, w)$. Note that $\Psi$ is continuous and compact. If we start from $\left(\beta^{0}, \alpha^{0}\right)=(\beta, \alpha)$, let $\left(\beta^{n+1}, \alpha^{n+1}\right)=\Psi\left(\beta^{n}, \alpha^{n}\right)$, for $n \geq 0$. We generate two sequences of functions, $\left\{\beta^{n}\right\}$ and $\left\{\alpha^{n}\right\}$ such that

$$
\beta \leq \beta^{1} \leq \cdots \leq \beta^{n} \leq \cdots \leq \alpha^{n} \leq \cdots \leq \alpha^{1} \leq \alpha
$$

on $I \cap \mathbb{T}$. Since $\left\{\beta^{n}\right\}$ and $\left\{\alpha^{n}\right\}$ are uniformly bounded, we then have two convergent subsequences $\left\{\beta^{n_{k}}\right\}$ and $\left\{\alpha^{n_{k}}\right\}$ with $\beta^{n_{k}} \rightarrow \beta_{*}$ and $\alpha^{n_{k}} \rightarrow \alpha^{*}$ uniformly on $I \cap \mathbb{T}$, as $n_{k} \rightarrow \infty$. By monotonicity of $\left\{\beta^{n}\right\}$ and $\left\{\alpha^{n}\right\}$, we see that $\beta^{n} \rightarrow \beta_{*}$ and $\alpha^{n} \rightarrow \alpha^{*}$ as $n \rightarrow \infty$. Thus we obtain $\Psi\left(\beta_{*}, \alpha^{*}\right)=\left(\beta_{*}, \alpha^{*}\right)$. By (B6), $\beta_{*} \equiv \alpha^{*}$ on $I \cap \mathbb{T}$. By (B5), we see that $\alpha^{*}$ is a solution of $(3.1),(3.2)$. We shall give some sufficient conditions for the existence of $F$ in Theorem 3.3 and Theorem 3.4 under some conditions. Consequently, we obtain the following results:

Theorem 3.5. Assume that there exist a positive constant $\varepsilon$ and two functions $\alpha, \beta \in C_{r d}^{1}\left(I \cap \mathbb{T}, \mathbb{R}^{N}\right)$ satisfying the following conditions :

(C1) $\alpha(t) \leq \beta(t)$ on $I \cap \mathbb{T}$,

(C2) for all $u \in C\left(I \cap \mathbb{T}, \mathbb{R}^{N}\right)$ with $\alpha \leq u \leq \beta$, we have

$$
\begin{gathered}
\alpha_{i}^{\Delta \Delta}+f_{i}(t, u) \geq-\frac{1}{2 \varepsilon}\left(u_{i}^{\sigma}-\alpha_{i}^{\sigma}\right) \\
\alpha(0)=\alpha\left(\sigma^{2}(T)\right), \quad \alpha^{\Delta}(0) \geq \alpha^{\Delta}(\sigma(T)) .
\end{gathered}
$$

(C3) for all $u \in C\left(I \cap \mathbb{T}, \mathbb{R}^{N}\right)$ with $\alpha \leq u \leq \beta$, we have

$$
\begin{gathered}
\beta_{i}^{\Delta \Delta}+f_{i}(t, u) \leq-\frac{1}{2 \varepsilon}\left(u_{i}^{\sigma}-\beta_{i}^{\sigma}\right) \\
\beta(0)=\beta\left(\sigma^{2}(T)\right), \quad \beta^{\Delta}(0) \leq \beta^{\Delta}(\sigma(T)) .
\end{gathered}
$$

(C4) $\left|f_{i}(t, u)-f_{i}(t, w)\right| \leq \frac{1}{\varepsilon}(u-w)$, for $u \geq w$.

Then the boundary value problem (3.1), (3.2) has a unique solution $u(t)$ with $\alpha(t) \leq$ $u(t) \leq \beta(t)$ on $I \cap \mathbb{T}$. 
Proof. Define

$$
F_{i}(t, u, v)=\frac{1}{2}\left[f_{i}(t, u)+f_{i}(t, v)+\frac{1}{2 \varepsilon}\left(u_{i}^{\sigma}-v_{i}^{\sigma}\right)\right] .
$$

It is trivial that (A5) holds. By (C2), (A2) is satisfied. And from (C3), (A3) is satisfied. (A4) is also true by (C4). Finally it remains to claim that (A6) is satisfied. If the assumption in (A6) holds. Let $V=\rho-\gamma$, then

$$
\begin{gathered}
V_{i}^{\Delta \Delta}-\frac{1}{2 \varepsilon} V_{i}^{\sigma}=0 \\
V(0)=V\left(\sigma^{2}(T)\right), \quad V^{\Delta}(0)=V^{\Delta}(\sigma(T)) .
\end{gathered}
$$

By Lemma 3.1, we have only the trivial solution, $V \equiv 0$ on $I \cap \mathbb{T}$. That is $\rho=\gamma$ on $I \cap \mathbb{T}$. By Theorem 3.3, the problem (3.1), (3.2) has a unique solution $u(t)$ with $\alpha(t) \leq u(t) \leq \beta(t)$ on $I \cap \mathbb{T}$.

Theorem 3.6. Assume that there exist two functions $\alpha, \beta \in C_{r d}^{1}\left(I \cap \mathbb{T}, \mathbb{R}^{N}\right)$ satisfying the following conditions:

(D1) $\beta(t) \leq \alpha(t)$ on $I \cap \mathbb{T}$,

(D2) for all $u \in C\left(I \cap \mathbb{T}, \mathbb{R}^{N}\right)$ with $\beta \leq u \leq \alpha$, we have

$$
\begin{gathered}
\alpha_{i}^{\Delta \Delta}+f_{i}(t, u) \geq \frac{k^{*}}{2}\left(u_{i}^{\sigma}-\alpha_{i}^{\sigma}\right) \\
\alpha(0)=\alpha\left(\sigma^{2}(T)\right), \quad \alpha^{\Delta}(0) \geq \alpha^{\Delta}(\sigma(T)) .
\end{gathered}
$$

where $k^{*}$ is given in Lemma 3.2 .

(D3) for all $u \in C\left(I \cap \mathbb{T}, \mathbb{R}^{N}\right)$ with $\beta \leq u \leq \alpha$, we have

$$
\begin{gathered}
\beta_{i}^{\Delta \Delta}+f_{i}(t, u) \leq \frac{k^{*}}{2}\left(u_{i}^{\sigma}-\beta_{i}^{\sigma}\right) \\
\beta(0)=\beta\left(\sigma^{2}(T)\right), \quad \beta^{\Delta}(0) \leq \beta^{\Delta}(\sigma(T)) .
\end{gathered}
$$

(D4) $\left|f_{i}(t, u)-f_{i}(t, w)\right| \leq \frac{k^{*}}{2}(w-u)$, for $u \geq w$.

Then the boundary value problem (3.1), (3.2) has a unique solution $u(t)$ with $\beta(t) \leq$ $u(t) \leq \alpha(t)$ on $I \cap \mathbb{T}$.

Proof. Define

$$
F_{i}(t, u, v)=\frac{1}{2}\left[f_{i}(t, u)+f_{i}(t, v)-\frac{k^{*}}{2}\left(u_{i}^{\sigma}-v_{i}^{\sigma}\right)\right] .
$$

Note that (B2) and (B3) are satisfied by (D2) and (D3) respectively. (B4) is satisfied by (D4), and (B5) is trival. (B6) is claimed as follows. If there exist two functions $\rho, \gamma \in C_{r d}^{1}\left(I \cap \mathbb{T}, \mathbb{R}^{N}\right)$ such that (3.37) and (3.38) hold, let $V=\rho-\gamma$, then

$$
\begin{gathered}
V_{i}^{\Delta \Delta}+\frac{k^{*}}{2} V_{i}^{\sigma}=0 \\
V(0)=V\left(\sigma^{2}(T)\right), V^{\Delta}(0)=V^{\Delta}(\sigma(T)) .
\end{gathered}
$$


By Lemma 3.2, we have only the trivial solution, $V \equiv 0$ on $I \cap \mathbb{T}$. That is $\rho=\gamma$ on $I \cap \mathbb{T}$ By Theorem 3.4, the problem (3.1), (3.2) has a unique solution $u(t)$ with $\beta(t) \leq u(t) \leq \alpha(t)$ on $I \cap \mathbb{T}$. The variants of Theorem 3.5 and Theorem 3.6 can be obtained analogously.

Theorem 3.7. Assume that there exist $F \in C\left(I \times \mathbb{R}^{N} \times \mathbb{R}^{N}, \mathbb{R}^{N}\right), \epsilon>0$ and two functions $\alpha, \beta \in C_{r d}^{1}\left(I, \mathbb{R}^{N}\right)$ satisfying the following conditions :

(E1) $\alpha(t) \leq \beta(t)$ on $I \cap \mathbb{T}$,

$$
\begin{gathered}
\alpha_{i}^{\Delta \Delta}+F_{i}(t, \alpha, \beta) \geq 0 \\
\alpha(0)=\alpha\left(\sigma^{2}(T)\right), \quad \alpha^{\Delta}(0) \geq \alpha^{\Delta}(\sigma(T)) .
\end{gathered}
$$

(E3)

$$
\begin{gathered}
\beta_{i}^{\Delta \Delta}+F_{i}(t, \beta, \alpha) \leq 0 \\
\beta(0)=\beta\left(\sigma^{2}(T)\right), \beta^{\Delta}(0) \leq \beta^{\Delta}(\sigma(T)) .
\end{gathered}
$$

(E4) $F_{i}(t, z, v)-F_{i}(t, u, v) \geq \frac{1}{\varepsilon}\left(z_{i}^{\sigma}-u_{i}^{\sigma}\right)$ for $z \geq u$, and $F_{i}(t, u, v) \leq F_{i}(t, u, w)$ for $v \geq w$.

(E5) $F_{i}(t, u, u)=f_{i}(t, u), 1 \leq i \leq N$.

(E6) If there exist two functions $\rho, \gamma \in C_{r d}^{1}\left(I \cap \mathbb{T}, \mathbb{R}^{N}\right)$ such that

$$
\begin{gathered}
\rho_{i}^{\Delta \Delta}+F_{i}(t, \rho, \gamma)=-\frac{1}{\varepsilon}\left(\gamma_{i}^{\sigma}-\rho_{i}^{\sigma}\right), \\
\left.\rho(0)=\rho\left(\sigma^{2}(T)\right), \quad \rho^{\Delta}(0)=\rho^{\Delta} \sigma(T)\right) .
\end{gathered}
$$

and

$$
\begin{gathered}
\gamma_{i}^{\Delta \Delta}+F_{i}(t, \gamma, \rho)=-\frac{1}{\varepsilon}\left(\rho_{i}^{\sigma}-\gamma_{i}^{\sigma}\right), \\
\gamma(0)=\gamma\left(\sigma^{2}(T)\right), \quad \gamma^{\Delta}(0)=\gamma^{\Delta}(\sigma(T)),
\end{gathered}
$$

then $\rho \equiv \gamma$ on $I \cap \mathbb{T}$.

Then the problem (3.1), (3.2) has a unique solution $u(t)$ with $\alpha(t) \leq u(t) \leq \beta(t)$ on $I \cap \mathbb{T}$.

Proof. For a pair $(\eta, \tau) \in[\alpha, \beta] \times[\alpha, \beta]$ with $\eta \leq \tau$, consider the linear boundary value problems :

$$
\begin{gathered}
u_{i}^{\Delta \Delta}-\frac{1}{\varepsilon} u_{i}^{\sigma}=-F_{i}(t, \eta, \tau)-\frac{1}{\varepsilon} \tau_{i}^{\sigma} \\
u(0)=u\left(\sigma^{2}(T)\right), \quad u^{\Delta}(0)=u^{\Delta}(\sigma(T)),
\end{gathered}
$$

and

$$
\begin{gathered}
w_{i}^{\Delta \Delta}-\frac{1}{\varepsilon} w_{i}^{\sigma}=-F_{i}(t, \tau, \eta)-\frac{1}{\varepsilon} \eta_{i}^{\sigma} \\
w(0)=w\left(\sigma^{2}(T)\right), \quad w^{\Delta}(0)=w^{\Delta}(\sigma(T)) .
\end{gathered}
$$

Let $V=u-w$, by (E4) and Lemma 3.1, we get $V \leq 0$ on $I \cap \mathbb{T}$. That is $u \leq w$ on $I \cap \mathbb{T}$. Similarly, by (E4) and Lemma 3.1 again, we also see that $w \leq \beta$ and $\alpha \leq u$ on $I \cap \mathbb{T}$. Thus there exists a unique solution $u$ of (3.47) and unique solution $w$ of (3.48) such that $\alpha \leq u \leq w \leq \beta$ on $I \cap \mathbb{T}$. Define a map $\Psi$ from $C\left(I \cap \mathbb{T}, R^{2 N}\right)$ into itself by 
$\Psi(\eta, \tau)=(u, w)$. Note that $\Psi$ is continuous and compact on $C\left(I \cap \mathbb{T}, R^{2 N}\right)$. If we start from $\left(\alpha^{0}, \beta^{0}\right)=(\alpha, \beta)$, let $\left(\alpha^{n+1}, \beta^{n+1}\right)=\Psi\left(\alpha^{n}, \beta^{n}\right), n \geq 0$. By the similar arguments as in the proof of Theorem 3.3, we obtain a non-decreasing sequence $\left\{\alpha^{n}\right\}$ and a nonincreasing sequence $\left\{\beta^{n}\right\}$ such that $\beta^{n} \rightarrow \beta_{*}$ and $\alpha^{n} \rightarrow \alpha^{*}$ as $n \rightarrow \infty$ for some $\beta_{*}$ and $\alpha^{*}$, and we also have $\left(\alpha^{*}, \beta_{*}\right)=\Psi\left(\alpha^{*}, \beta_{*}\right)$. By (E6), $\beta_{*} \equiv \alpha^{*}$ on $I \cap \mathbb{T}$. Thus by (E5), we get the solution of (3.1), (3.2).

Theorem 3.8. Assume that there exist $F \in C\left(I \times \mathbb{R}^{N} \times \mathbb{R}^{N}, \mathbb{R}^{N}\right)$, and two functions $\alpha, \beta \in C_{r d}^{1}\left(I, \mathbb{R}^{N}\right)$ satisfying the following conditions :

(F1) $\beta(t) \leq \alpha(t)$ on $I \cap \mathbb{T}$,

(F3)

$$
\begin{gathered}
\alpha_{i}^{\Delta \Delta}+F_{i}(t, \alpha, \beta) \geq 0 \\
\alpha(0)=\alpha\left(\sigma^{2}(T)\right), \quad \alpha^{\Delta}(0) \geq \alpha^{\Delta}(\sigma(T)) .
\end{gathered}
$$

$$
\begin{gathered}
\beta_{i}^{\Delta \Delta}+F_{i}(t, \beta, \alpha) \leq 0 \\
\beta(0)=\beta\left(\sigma^{2}(T)\right), \beta^{\Delta}(0) \leq \beta^{\Delta}(\sigma(T)) .
\end{gathered}
$$

(F4) $F_{i}(t, z, v)-F_{i}(t, u, v) \leq k^{*}\left(z_{i}^{\sigma}-u_{i}^{\sigma}\right)$ for $z \geq u$, where $k^{*}$ is given in Lemma 3.2 and $F_{i}(t, u, v) \geq F_{i}(t, u, w)$ for $v \geq w$.

(F5) $F_{i}(t, u, u)=f_{i}(t, u), 1 \leq i \leq N$.

(F6) If there exist two functions $\rho, \gamma \in C_{r d}^{1}\left(I \cap \mathbb{T}, \mathbb{R}^{N}\right)$ such that

$$
\begin{aligned}
\rho_{i}^{\Delta \Delta}+F_{i}(t, \rho, \gamma) & =0 \\
\rho(0)=\rho\left(\sigma^{2}(T)\right), \quad \rho^{\Delta}(0) & =\rho^{\Delta}(\sigma(T)),
\end{aligned}
$$

and

$$
\begin{gathered}
\gamma_{i}^{\Delta \Delta}+F_{i}(t, \gamma, \rho)=0 \\
\gamma(0)=\gamma\left(\sigma^{2}(T)\right), \quad \gamma^{\Delta}(0)=\gamma^{\Delta}(\sigma(T)) .
\end{gathered}
$$

then $\rho \equiv \gamma$ on $I \cap \mathbb{T}$.

Then the problem (3.1), (3.2) has a unique solution $u(t)$ with $\beta(t) \leq u(t) \leq \alpha(t)$ on $I \cap \mathbb{T}$.

Proof. For a pair $(\eta, \tau) \in[\beta, \alpha] \times[\beta, \alpha]$ with $\eta \leq \tau$, consider the linear boundary value problems :

$$
\begin{gathered}
u_{i}^{\Delta \Delta}+k^{*} u_{i}^{\sigma}=-F_{i}(t, \eta, \tau)+k^{*} \eta_{i}^{\sigma} \\
u(0)=u\left(\sigma^{2}(T)\right), \quad u^{\Delta}(0)=u^{\Delta}(\sigma(T)),
\end{gathered}
$$

and

$$
\begin{gathered}
w_{i}^{\Delta \Delta}+k^{*} w_{i}^{\sigma}=-F_{i}(t, \tau, \eta)+k^{*} \tau_{i}^{\sigma} \\
w(0)=w\left(\sigma^{2}(T)\right), \quad w^{\Delta}(0)=w^{\Delta}(\sigma(T)) .
\end{gathered}
$$

Now for $V=w-u$, we have

$$
\begin{gathered}
V_{i}^{\Delta \Delta}+k^{*} V_{i}^{\sigma}=F_{i}(t, \eta, \tau)-F_{i}(t, \tau, \eta)+k^{*}\left(\tau_{i}^{\sigma}-\eta_{i}^{\sigma}\right) \\
V(0)=V\left(\sigma^{2}(T)\right), \quad V^{\Delta}(0)=V^{\Delta}(\sigma(T)) .
\end{gathered}
$$


By (F4), the right hand side of the equation is nonnegative. By lemma 3.2, we get $V \geq 0$ on $I \cap \mathbb{T}$. That is $w \geq u$ on $I \cap \mathbb{T}$. Also we see that $u \geq \beta$ on $I \cap \mathbb{T}$. In fact, let $W=u-\beta$ on $I \cap \mathbb{T}$, we have

$$
W_{i}^{\Delta \Delta}+k^{*} W_{i}^{\sigma} \geq F_{i}(t, \beta, \alpha)-F_{i}(t, \eta, \tau)+k^{*}\left(\eta_{i}^{\sigma}-\beta_{i}^{\sigma}\right) .
$$

By (F4), we then have

$$
\begin{gathered}
W_{i}^{\Delta \Delta}+k^{*} W_{i}^{\sigma} \geq 0 \\
W(0)=W\left(\sigma^{2}(T)\right), \quad W^{\Delta}(0) \geq W^{\Delta}(\sigma(T)) .
\end{gathered}
$$

By lemma 3.2 again, we have $u \geq \beta$ on $I \cap \mathbb{T}$. Similarly we have $\alpha \geq w$ on $I \cap \mathbb{T}$. Thus there exists a unique solution $u$ of (3.49) and unique solution $w$ of (3.50) such that $\alpha \geq w \geq u \geq \beta$ on $I \cap \mathbb{T}$. Define a map $\Psi$ from $C\left(I \cap \mathbb{T}, R^{2 N}\right)$ into itself by $\Psi(\eta, \tau)=(u, w)$. Note that $\Psi$ is continuous and compact on $C\left(I \cap \mathbb{T}, R^{2 N}\right)$. If we start from $\left(\beta^{0}, \alpha^{0}\right)=(\beta, \alpha)$, let $\left(\beta^{n+1}, \alpha^{n+1}\right)=\Psi\left(\beta^{n}, \alpha^{n}\right), n \geq 0$. By the similar arguments as in the proof of Theorem 3.3, a non-increasing sequence $\left\{\beta^{n}\right\}$ and a non-decreasing sequence $\left\{\alpha^{n}\right\}$ are obtained such that $\beta^{n} \rightarrow \beta_{*}$ and $\alpha^{n} \rightarrow \alpha^{*}$ as $n \rightarrow \infty$ for some $\beta_{*}$ and $\alpha^{*}$, and we also have $\left(\beta_{*}, \alpha^{*}\right)=\Psi\left(\beta_{*}, \alpha^{*}\right)$. By (F6), $\beta_{*} \equiv \alpha^{*}$ on $I \cap \mathbb{T}$. Thus by (F5), we get the solution of (3.1), (3.2).

Theorem 3.9. Assume that there exist two functions $\alpha, \beta \in C_{r d}^{1}\left(I \cap \mathbb{T}, \mathbb{R}^{N}\right)$ satisfying the following conditions :

(G1) $\alpha(t) \leq \beta(t)$ on $I \cap \mathbb{T}$,

$$
\begin{gathered}
\alpha_{i}^{\Delta \Delta}+f_{i}(t, \alpha) \geq 0 \\
\alpha(0)=\alpha\left(\sigma^{2}(T)\right), \quad \alpha^{\Delta}(0) \geq \alpha^{\Delta}(\sigma(T)) . \\
\beta_{i}^{\Delta \Delta}+f_{i}(t, \beta) \leq 0 \\
\beta(0)=\beta\left(\sigma^{2}(T)\right), \quad \beta^{\Delta}(0) \leq \beta^{\Delta}(\sigma(T)) .
\end{gathered}
$$

(G4) $f_{i}(t, w)-f_{i}(t, u) \geq \frac{1}{\varepsilon}\left(w_{i}^{\sigma}-u_{i}^{\sigma}\right)$ for $w \geq u$ and for some $\varepsilon>0$.

Then the boundary value problem (3.1), (3.2) has a unique solution $u(t)$ with $\alpha(t) \leq$ $u(t) \leq \beta(t)$ on $I \cap \mathbb{T}$.

Proof. Let

$$
F_{i}(t, u, v)=\frac{1}{2}\left[f_{i}(t, u)+f_{i}(t, v)+\frac{1}{\varepsilon}\left(u_{i}^{\sigma}-v_{i}^{\sigma}\right)\right] .
$$

Then by (G2) and (G4), we have (E2). And by (G3) and (G4), (E4) is satisfied. (E5) is trivial by the definition. (E6) is claimed as follows. Suppose that there exist two functions $\rho$ and $\gamma$ such that

$$
\begin{gathered}
\rho_{i}^{\Delta \Delta}+\frac{1}{2}\left[f_{i}(t, \rho)+f_{i}(t, \gamma)+\frac{1}{\varepsilon}\left(\rho_{i}^{\sigma}-\gamma_{i}^{\sigma}\right)\right]=-\frac{1}{\varepsilon}\left(\gamma_{i}^{\sigma}-\rho_{i}^{\sigma}\right), \\
\rho(0)=\rho\left(\sigma^{2}(T)\right), \quad \rho^{\Delta}(0)=\rho^{\Delta}(\sigma(T)),
\end{gathered}
$$


and

$$
\begin{gathered}
\gamma_{i}^{\Delta \Delta}+\frac{1}{2}\left[f_{i}(t, \gamma)+f_{i}(t, \rho)+\frac{1}{\varepsilon}\left(\gamma_{i}^{\sigma}-\rho_{i}^{\sigma}\right)\right]=-\frac{1}{\varepsilon}\left(\rho_{i}^{\sigma}-\gamma_{i}^{\sigma}\right), \\
\gamma(0)=\gamma\left(\sigma^{2}(T)\right), \quad \gamma^{\Delta}(0)=\gamma^{\Delta}(\sigma(T)) .
\end{gathered}
$$

Let $V=\rho-\gamma$, we get

$$
V_{i}^{\Delta \Delta}-\frac{1}{\varepsilon} V_{i}^{\sigma}=0, \quad V(0)=V\left(\sigma^{2}(T)\right), \quad V^{\Delta}(0)=V^{\Delta}(\sigma(T)) .
$$

By Lemma 3.1, $V=0$ on $I \cap \mathbb{T}$, that is $\rho \equiv \gamma$ on $I \cap \mathbb{T}$. By Theorem 3.7, problem (3.1), (3.2) has a unique solution $u$ on $I \cap \mathbb{T}$.

Theorem 3.10. Assume that there exist two functions $\alpha, \beta \in C_{r d}^{1}\left(I \cap \mathbb{T}, \mathbb{R}^{N}\right)$ satisfying the following conditions :

(H1) $\beta(t) \leq \alpha(t)$ on $I \cap \mathbb{T}$,

(H2)

$$
\begin{gathered}
\alpha_{i}^{\Delta \Delta}+f_{i}(t, \alpha) \geq 0 \\
\alpha(0)=\alpha\left(\sigma^{2}(T)\right), \quad \alpha^{\Delta}(0) \geq \alpha^{\Delta}(\sigma(T)) .
\end{gathered}
$$

$$
\begin{gathered}
\beta_{i}^{\Delta \Delta}+f_{i}(t, \beta) \leq 0 \\
\beta(0)=\beta\left(\sigma^{2}(T)\right), \quad \beta^{\Delta}(0) \leq \beta^{\Delta}(\sigma(T)) .
\end{gathered}
$$

(H4) $f_{i}(t, w)-f_{i}(t, u) \leq k^{*}\left(w_{i}^{\sigma}-u_{i}^{\sigma}\right)$ for $w \geq u$ and $k^{*}$ is given in Lemma 3.2 .

Then the boundary value problem (3.1), (3.2) has a unique solution $u(t)$ with $\beta(t) \leq$ $u(t) \leq \alpha(t)$ on $I \cap \mathbb{T}$.

Proof. Let

$$
F_{i}(t, u, v)=\frac{1}{2}\left[f_{i}(t, u)+f_{i}(t, v)+k^{*}\left(u_{i}^{\sigma}-v_{i}^{\sigma}\right)\right] .
$$

Then by (H2) and (H4), we have

$$
\alpha_{i}^{\Delta \Delta}+F_{i}(t, \alpha, \beta) \geq \alpha_{i}^{\Delta \Delta}+f_{i}(t, \alpha) \geq 0,
$$

and similarly, by (H3) and (H4), (F3) is satisfied. By (H4), (F4) is also true and (F5) is trivial by the definition of $F$. (F6) is shown as follows. Suppose that there exist two functions $\rho$ and $\gamma$ such that

$$
\begin{gathered}
\rho_{i}^{\Delta \Delta}+\frac{1}{2}\left[f_{i}(t, \rho)+f_{i}(t, \gamma)+k^{*}\left(\rho_{i}^{\sigma}-\gamma_{i}^{\sigma}\right)\right]=0 \\
\rho(0)=\rho\left(\sigma^{2}(T)\right), \quad \rho^{\Delta}(0)=\rho^{\Delta}(\sigma(T)),
\end{gathered}
$$

and

$$
\begin{gathered}
\gamma_{i}^{\Delta \Delta}+\frac{1}{2}\left[f_{i}(t, \gamma)+f_{i}(t, \rho)+k^{*}\left(\gamma_{i}^{\sigma}-\rho_{i}^{\sigma}\right)\right]=0 \\
\gamma(0)=\gamma\left(\sigma^{2}(T)\right), \quad \gamma^{\Delta}(0)=\gamma^{\Delta}(\sigma(T)) .
\end{gathered}
$$


Let $V=\rho-\gamma$, we get

$$
V_{i}^{\Delta \Delta}+k^{*} V_{i}^{\sigma}=0, \quad V(0)=V\left(\sigma^{2}(T)\right), \quad V^{\Delta}(0)=V^{\Delta}(\sigma(T)) .
$$

By Lemma 3.2, $V=0$ on $I \cap \mathbb{T}$, that is $\rho \equiv \gamma$ on $I \cap \mathbb{T}$. By Theorem 3.8, problem (3.1), (3.2) has a unique solution $u$ on $I \cap \mathbb{T}$.

\section{References}

[1] R. P. Agarwal and M. Bohner, Quadratic functionals for second order matrix equations on time scales, Nonlinear Anal., 33 (1988), 675-692.

[2] E. Akin, Boundary value problems for a differential equations on a measure chain, Panamerican Math. J., 10(2000), 17-30.

[3] B. Aulbach and S. Hilger, A unified approach to continuous and discrete dynamics, In Differential Equations : Qualitative Theory, Colloq. Math. Soc. János Bolyai, North-Holland, (1988), 37-57.

[4] M. Bohner, S. Clark and J. Ridenhour, Lyapunov inequalities on time scales, Math. Inequal. \& Appl., 4(2001), 535-557.

[5] M. Bohner and A. Peterson, Dynamic Equations on Time Scales, Birkhäuser, 2001.

[6] C. de Coster and P. Habets, Upper and lower solutions in the theory of ODE boundary value problems: classical and recent results, Nonlinear analysis and boundary value problems for ordinary differential equations, (edited by F. Zanolin), C.I.S.M. Courses and Lectures, vol. 371, Springer-Verlag, 1993, 1-79.

[7] S. Hilger, Analysis on measure chain - a unified approach to continuous and discrete calculus, Res. in Math., 18 (1990), 18-56.

[8] R. Kannan and V. Lakshmikantham, Existence of periodic solutions of nonlinear boundary value problems and the method of upper and lower solutions, Appl. Anal., 17(1984), 103113.

[9] G. S. Ladde, V. Lakshmikantham and A.S. Vatsala, Monotone Iterative Techniques for Nonlinear Differential Equations, Pitman Press, 1985.

[10] V. Lakshmikatham, V. S. Sivasundaran and B. Kaymakcalan, Dynamic Systems on Measure Chains, Kluwer Academic Publishers, Netherlands, 1996.

General Education Center, National Taipei University of Technology, Taipei, Taiwan 106.

E-mail: stwu@ntut.edu.tw

Department of Mathematical Science, National Chengchi University, Taipei, TAIWAN 116. 